\title{
Computer simulation of colloid-polymer mixtures
}

\author{
Evert Jan Meijer ${ }^{1}$, Daan Frenkel \\ FOM Institute for Atomic and Molecular Physics, Kruislaan 407, 1098 SJ Amsterdam, The Netherlands
}

\begin{abstract}
We present results of a computer simulation study of a simple model for a colloid dispersed in a dilute polymer solution. The study aims to investigate the role of entropy in such mixtures. We have computed the polymer-induced interaction, the phase behaviour, and the local structure of the colloidal component, and determined the dependence of these properties on the polymer-colloid size ratio.
\end{abstract}

\section{Introduction}

The addition of non-adsorbing polymer to a colloidal dispersion gives rise to a change in the properties of the colloid. The main feature observed is a coagulation of the colloidal particles upon adding a sufficient amount of polymer [1,2]. This effect is due to the fact that the presence of the polymers induces an attraction between the colloidal particles [3,4]. This attraction has an entropic origin: Due to the finite extent of the polymers, there is a region around the colloidal particles that is not, or less, accessible to the polymers. In this region the polymer concentration is depleted. The depletion layers around the colloidal particles will overlap if the colloidal particles approach each other. This gives rise to a decrease of the total volume of the regions with a depleted polymer concentration, or, equivalently, an increase of the total volume accessible to the polymers. Hence the entropy of the polymers will increase upon decreasing distance between the colloidal particles. This effect induces an (entropic) attraction, that is usually referred to as the polymer-induced depletion force. An important feature of the polymer-induced interaction is the fact that it is, in general, not pair-wise additive in the coordinates of the colloidal particles.

\footnotetext{
1 Present address: Statistical Physics of Condensed Matter, Université Libre de Bruxelles, CP223, Campus Plaine, Bld. du Triomphe, B-1050 Bruxelles, Belgium.
} 
Recently, we have studied the role of entropy in colloid-polymer mixtures by means of computer simulation of a simple model [5-8]. In the following we will summarize some of the main results that were obtained in this study, and present some recent results for the local structure of the colloid. For a comprehensive description of the model, the numerical techniques, the simulation results, and a comparison with theoretical predictions, we refer to Ref. [8].

\section{Model}

In the present model the colloidal particles are represented by hard spheres with a diameter $\sigma$. The polymers are modeled by (non-self-avoiding) ideal chains, confined to a cubic lattice, that have an excluded volume interaction with the colloidal hard spheres. The length of a polymer, i.e. the number of connected lattice links that constitute the polymer, is denoted by $\ell$. We will refer to this model as the lattice-polymer model. In the following we will estimate the size of the lattice polymer by its radius-of-gyration $R_{g}$, that is approximately $R_{g} \sim \sqrt{\ell / 6}$. Energies are expressed in units of $k_{B} T$, where $k_{B}$ denotes the Boltzmann constant, and $T$ the temperature.

The partition function $\Xi$ of a lattice-polymer model of $N$ colloidal particles in chemical equilibrium with a polymer solution of fugacity $z$ is given by [5]:

$$
\Xi(N, z, V)=\int_{V} \mathrm{~d} r^{N} \exp \left[-U_{h s}\left(\boldsymbol{r}^{N}\right)+z \Omega\left(\boldsymbol{r}^{N} ; V\right)\right] .
$$

Here, $V$ denotes the volume, $r^{N}$ the coordinates of the $N$ colloidal particles, $U_{h s}$ the hard-sphere interaction, and $\Omega$ the partition function of a single polymer. The latter yields the number of accessible polymer conformation, and is also referred to as the free volume. Eq. (1) shows that the colloid-polymer mixture can be considered a onecomponent system with an interaction potential $U_{c o l+p o l}$, that is a combination of a hard-sphere repulsion $U_{h s}$, and a polymer-induced interaction $z \Omega$ :

$$
U_{c o l+p o l}\left(\boldsymbol{r}^{N}\right)=U_{h s}\left(\boldsymbol{r}^{N}\right)-z \Omega\left(\boldsymbol{r}^{N} ; V\right) .
$$

As mentioned above, the polymer-induced interaction is attractive, and, except for the shortest polymers, not pair-wise additive in the colloidal coordinates. To illustrate the latter point it is convenient to consider the number of non-accessible conformations, denoted by $\bar{\Omega}$. This number is the exact measure for total volume of the depletion region. Clearly, for sufficiently long polymers there will be, in addition to polymer conformations that intersect with two colloidal particles, conformations intersecting with three colloidal particles. Hence, the total number of excluded conformations, and consequently the polymer-induced interaction, depends on the positions of triplets of colloidal particles. For an illustration see Fig. 1.

The thermodynamic properties of the lattice-polymer model can be deduced from the free energy $F=-\ln \Xi$. The free energy can be obtained by thermodynamic integration using the hard-sphere system as a reference: 

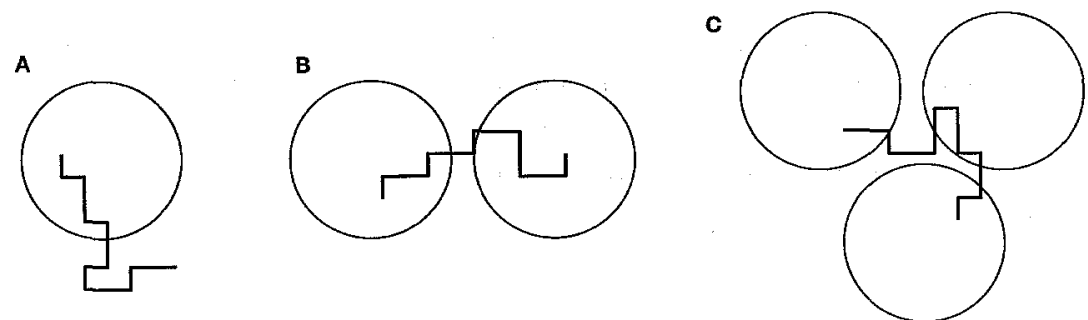

Fig. 1. One-, two-, and three-body contributions to the total number of excluded conformations $\bar{\Omega}$. The circles indicate the hard-sphere colloidal particles. The lines represent the polymer confined to cubic lattice. (A) illustrates the one-body contribution. (B) illustrates the two-body contribution (denoted by $\bar{\Omega}^{2 b}$ ), that corrects for the doubly counted conformations by the one-body contributions. (C) illustrates the three-body contribution (denoted by $\bar{\Omega}^{3 b}$ ), that corrects for the doubly counted conformations in the sum of the one- and two-body contributions.

$$
F(N, z, V)=F_{h s}(N, V)-\int_{0}^{z} \mathrm{~d} z\langle\Omega\rangle_{N, z, V} .
$$

$F_{h s}$ denotes the hard-sphere free energy, and is known accurately from simulation $[9,10]$. Hence, the study of the polymer-induced interactions (Eq. (2)), and the thermodynamic properties (Eq. (3)) of the model colloid-polyer mixture deduces to the computation of the (average) free volume $(<\Omega>$ ) as function of the colloid density $\rho=N / V$, and the polymer fugacity $z$.

The free volume $\Omega$ of a lattice polymer can be computed using an efficient lattice algorithm, that is based on the correspondence between an ideal polymer and diffusion in a lattice gas [5]. Properties of the model colloid-polymer mixture, such as the average free volume $\langle\Omega\rangle$, or the local structure of the colloid, can be determined using standard Monte-Carlo techniques for the simulation of a one-component system with an interaction potential $U_{c o l+p o l}$ (Eq. (2)) $[6,8]$.

\section{Simulation results}

The polymer-induced interaction $z \Omega$ arises from the exclusion of polymer conformations by two, or more colloidal particles simultaneously. The number of conformations excluded by two, and three colloidal particles simultaneously yield the two- and threebody contribution to the polymer-induced interaction. The relative importance of these two contributions depends strongly on the polymer-colloid size ratio. This is illustrated in Fig. (2), that shows computer simulation results [6] for the two- and three-body contribution in the lattice-polymer model for 'short' $\left(2 R_{g} / \sigma \sim 0.25\right)$, and 'long' $\left(2 R_{g} / \sigma \sim\right.$ $0.55)$ polymers. Clearly, for the 'short' polymers the repulsive three-body contribution is negligible compared to the attractive two-body contribution. In contrast, for the 'long' polymers the three-body contribution is a significant fraction of the two-body contribution. Simulations of the lattice-polymer model have shown that the non-pair-wise 

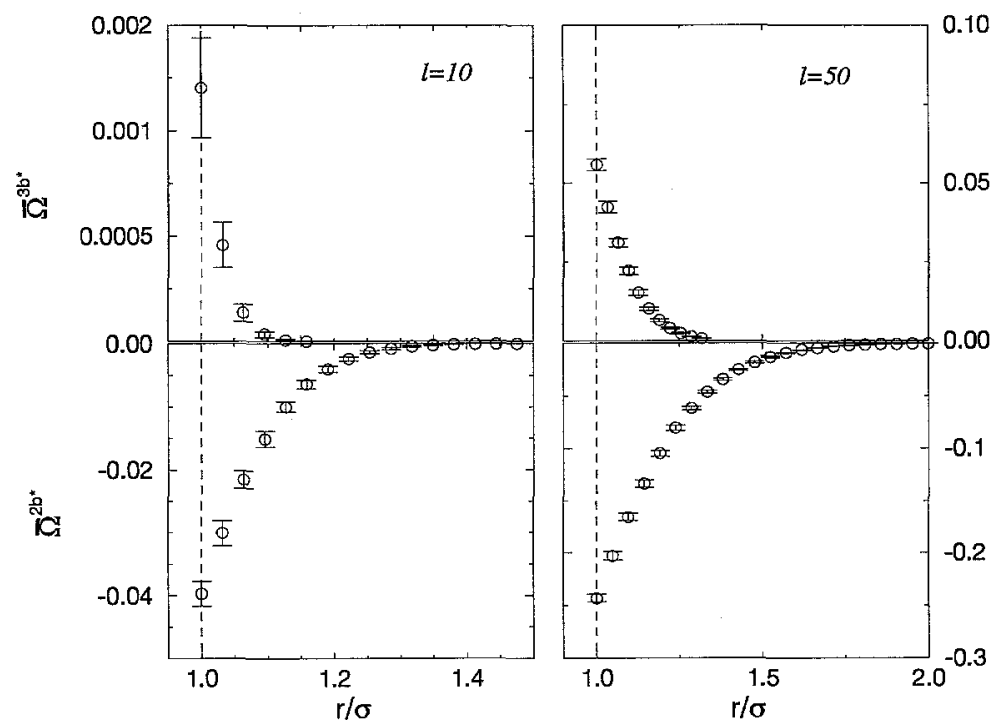

Fig. 2. Simulation results for the two- and three-body contribution (see caption Fig. 1) to the number of excluded conformations $\bar{\Omega}$ as function of the distance $r$ between the colloidal spheres $(\sigma=10.5)$. The two figures show the results for 'short' $(\ell=10)$ and 'long' $(\ell=50)$ polymers. The lower part of each figure gives the results for the normalized (attractive) two-body contribution $\left(\bar{\Omega}^{2 b *}\right)$. The upper part shows the results for the normalized (repulsive) three-body contributions $\left(\bar{\Omega}^{3 b *}\right)$, where the three colloidal spheres were configurated with their centers on an equilateral triangle. The circles indicate simulation results for the lattice-polymer model, and the error-bars show the mean-square variation in the computed values, due to the discreteness of the polymer lattice. (Results from Ref. [6].)

additivity of the polymer-induced interaction has a pronounced effect on both the local structure [6] and the phase diagram [7,8] of a colloid-polymer mixture. This indicates that in the theoretical studies of these systems the non-pair-wise additivity of the polymer-induced interaction should be taken into account.

The phase behaviour of the colloidal component has been subject of extensive experimental (see e.g. Refs. [11-14]) and theoretical (see e.g. Refs. [15-17]) study. We recall, that the (reference) hard-sphere system exhibits a disordered fluid-like phase at low densities $\left(\rho \sigma^{3} \leq 0.94 ; \rho=N / V\right)$ and an ordered solid-like phase at high densities $\left(\rho \sigma^{3} \geq 1.04\right)$. The studies have indicated that the addition of polymer to a hard-sphere-like system induces a change in the location of the fluid-solid coexistence region. Moreover, they have shown that for sufficiently long polymers, the colloidal fluid phase-separates into a dilute vapour-like phase and a dense liquid-like phase. To illustrate the effect of the polymer-colloid size ratio on the phase diagram we show in Figs. 3 and 4 simulation results [8] of the phase diagram of the lattice-polymer model with 'short' $\left(2 R_{g} / \sigma=0.25\right)$ and with 'long' $\left(2 R_{g} / \sigma=0.55\right)$ polymers, respectively. The phase-boundaries are determined by absolute free-energy calculations of the various phases involved. Clearly, the two phase diagrams are of a different type. In the mixture with the 'long' polymers (Fig. 4), the colloidal fluid phase-separates into a vapour-like 


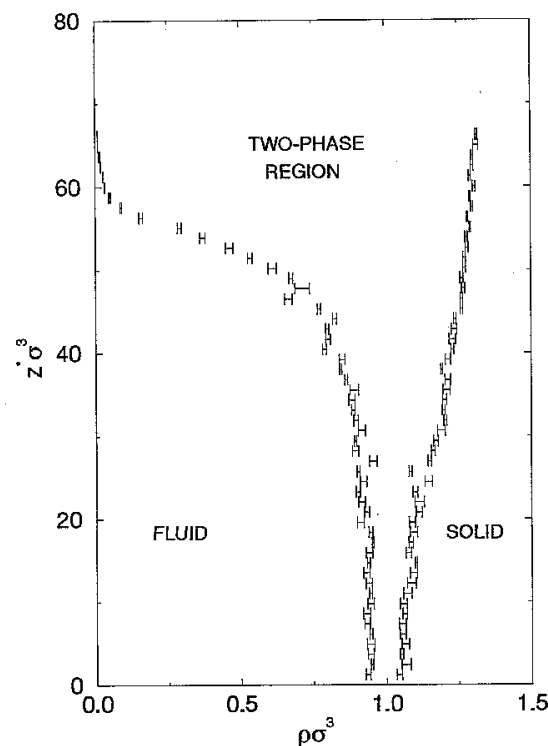

Fig. 3

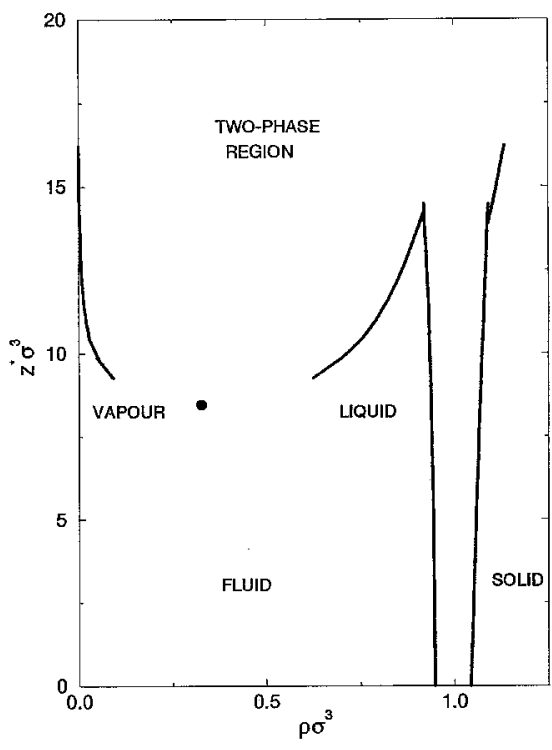

Fig. 4

Fig. 3. Phase diagram, obtained by Monte Carlo simulation, of lattice-polymer model $(\sigma=10.5)$ with 'short' polymers of length $\ell=10$. The points with error bars indicate the reduced densities $\rho \sigma^{3}$ of the coexisting colloidal phases as function of the reduced polymer fugacity $z^{*} \sigma^{3}\left(z^{*}=z \times\left(6^{\ell} / 2\right)\right)$. (Results from Ref. [8].)

Fig. 4. Phase diagram, obtained by Monte Carlo simulation, of lattice-polymer model $(\sigma=10.5)$ with 'long' polymers of length $\ell=50$, obtained by Monte Carlo simulation. The draw lines show the reduced densities $\rho \sigma^{3}$ of the coexisting colloidal phases as function of the reduced polymer fugacity $z^{*} \sigma^{3}\left(z^{*}=z \times\left(6^{\ell} / 2\right)\right)$. The black circle indicates the estimated critical point. (Results from Ref. [8].)

and a liquid-like phase, above a 'critical' fugacity. Above a 'triple' polymer fugacity the liquid-like phase disappears: there remains an equilibrium between a very dilute colloidal vapour-like phase and a colloidal solid. Here, the 'critical'- and 'triple' fugacity are analogous to the inverse critical- and triple-temperature in simple molecular fluids. In contrast, in the colloid-polymer mixture with the 'short' polymers (Fig. 3) the colloid exhibits only a 'fluid-solid'-like coexistence. Apparently, the stability of a 'liquid'-like colloidal phase in a colloid-polymer mixture requires sufficiently long polymers: the ratio of the ranges of attractive polymer-induced interaction and the repulsive colloidal hard-sphere interaction should be sufficiently large. For the lattice-polymer model we estimate the minimal ratio to be $2 R_{g} / \sigma=0.45$ [8].

The local structure of a colloid is one of the properties that can be probed directly by scattering techniques. We have computed the radial distribution function $g(r)$ and the structure function $S(k)$ of the the colloidal component in the lattice-polymer model. These results have been determined by Monte Carlo simulation of a $N=256$ colloid system, using standard analyzing methods $[18,19]$. They may serve as a reference for comparison with experimental data, that have been, to our knowledge, not widely 


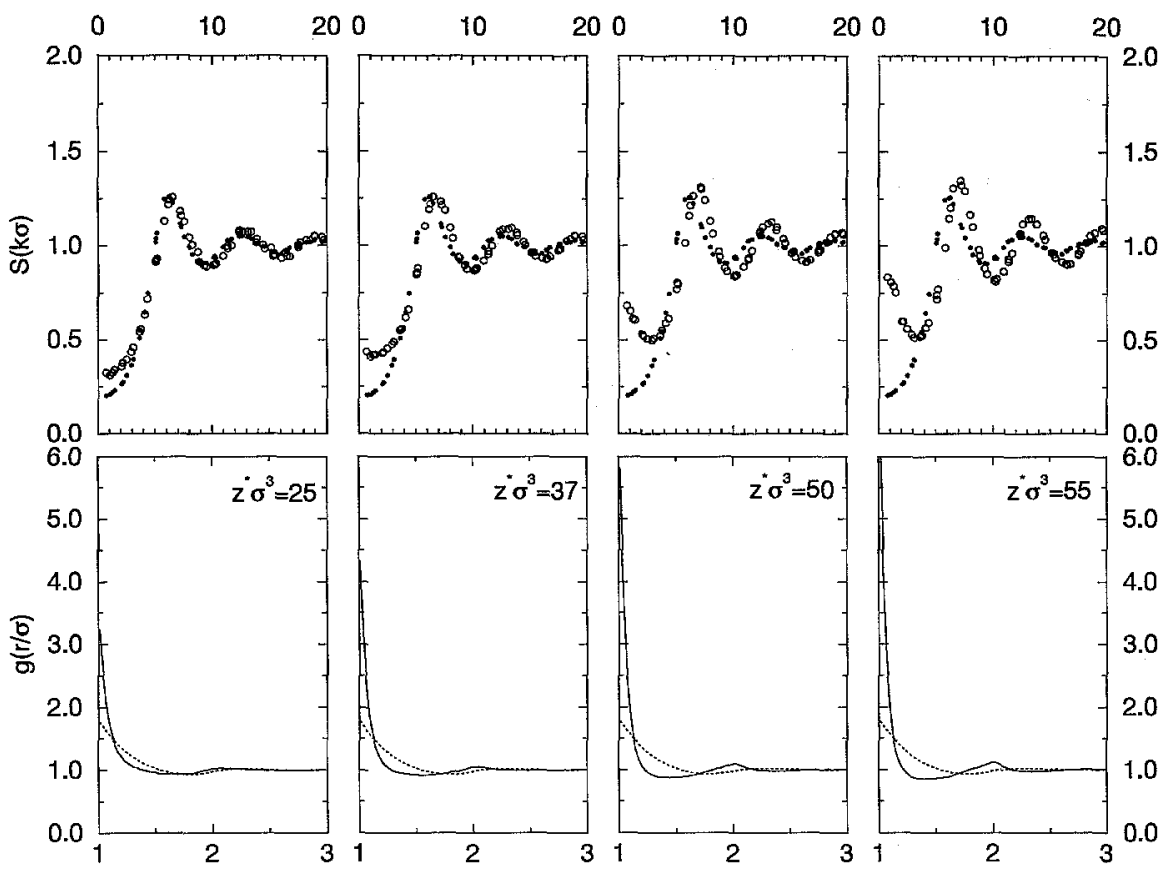

Fig. 5. Simulation results for the radial distribution function $g(r / \sigma)$ and structure function $S(k \sigma)$ of the colloidal particles in the lattice-polymer model with polymers of length $\ell=10$ and colloidal particles of diameter $\sigma=10.5$. The figure shows results for various values of the polymer fugacity $z^{*} \sigma^{3}\left(z^{*}=z \times\left(6^{\ell} / 2\right)\right)$ up to the coexistence line (see Fig. 3), at a colloid density $\rho \sigma^{3}=0.40$. In the plots of $S(k \sigma)$ the open circles denote the results for the colloid-polymer mixture, and the black dots the results for a pure hard-sphere fluid, that are given for reference. In the plots of $g(r / \sigma)$ the drawn line indicates the results for the colloid-polymer mixture, and the dotted line the results for a hard-sphere fluid.

reported yet. Fig. 5 shows the simulation results for a colloid-polymer mixture with 'short' polymers $(\ell=10 ; \sigma=10.5)$ at a colloid density $\rho \sigma^{3}=0.40$, for several polymer fugacities up to the coexistence line (see Fig. 3). Clearly, with increasing polymer fugacity the structure deviates increasingly from the hard-sphere structure. In particular, the contact value of $g(r)$ is significantly enhanced. Close to the coexistence line $\left(z^{*} \sigma^{3} \sim 50\right)$ the shape of $S(k)$ has changed markedly with respect to shape of the hard-sphere $S(k)$. Moreover we observe that the location of the peaks of $S(k)$ shift to higher values of $k$.

\section{Summary}

This paper briefly describes some recent results of a computer-simulation study of a simple excluded-volume model for a colloid dispersed in a dilute polymer solution. This study aimed to determine the equilibrium properties and to investigate the role entropy plays in colloid-polymer mixtures. Results for the polymer-induced interaction, the phase diagram, and the local structure of the colloidal component are presented. 
The polymer-induced interaction is, except for the shortest polymers, not pair-wise additive in the colloidal coordinates. This has a significant influence on properties such as the phase diagram and the local structure of the colloid. The phase diagram of the colloidal component shows a 'fluid-solid' equilibrium in mixtures with 'short' polymers. In a mixture with 'long' polymers an additional 'vapour-liquid' equilibrium appears for fugacities above a 'critical' value and below a 'triple' value. The local structure of the colloidal component changes significantly with increasing polymer concentration, in particular close to the coexistence line.

In this work the polymer-polymer interactions have been neglected. These are expected to affect the properties at high polymer fugacities. Presently we are employing an extension of the numerical techniques applied in the work presented here, that treats the polymer-polymer interactions in a mean-field approximation [20].

\section{Acknowledgement}

We thank A. Vrij, H. Lekkerkerker, and B.M. Mulder for useful suggestions and discussions. This work is part of the research program of the Stichting voor Fundamenteel Onderzoek der Materie (Foundation for Fundamental Research of Matter) and was made possible by financial support from the Nederlandse Organisatie voor Wetenschappelijk Onderzoek (Netherlands Organisation for the Advancement of Research), and the Dutch Ministry of Economic Affairs within the framework of the Innovatief Onderzoeksprogramma Polymeercomposieten en Bijzondere Polymeren (Innovative Research Program Polymer-composites and Special Polymers). Computer time on the CRAY-YMP at SARA, was made available through support of the Stichting Computer Faciliteiten (Foundation for National Computing Facilities).

\section{References}

[1] B. R. Monoghan and H. L. White, J. Gen. Physiol. 19 (1935) 715.

[2] C. Bondy, Trans. Faraday Soc. 35 (1939) 1093.

[3] S. Asakura and F. Oosawa, J. Chem. Phys. 22 (1954) 1255.

[4] A. Vrij, Pure Appl. Chem. 48 (1976) 471.

[5] D. Frenkel, J. Phys.: Condens. Matter 2 (1990) SA265.

[6] E. J. Meijer and D. Frenkel, Phys. Rev. Lett. 67 (1991) 1110.

[7] E. J. Meijer and D. Frenkel, Tenside Surf. Det. 30 (1993) 269.

[8] E. J. Meijer and D. Frenkel, J. Chem. Phys. 100 (1994) 6873.

[9] W. G. Hoover and F. H. Ree, J. Chem. Phys. 49 (1968) 3609.

[10] K. R. Hall, J. Chem. Phys. 57 (1972) 2252.

[11] H. de Hek and A. Vrij, J. Colloid Interface Sci. 84 (1981) 409.

[12] B. Vincent, J. Edwards, S. Emmett, and R. Groot, Colloid Surf. 31 (1988) 267.

[13] W. C. K. Poon, J. S. Selfe, M. B. Robertson, S. M. Ilett, A. D. Pirie and P. N. Pusey, J. Phys. II France 3 (1993) 1075.

[14] R. L. Calderon, J. Bibette and J. Biais, Europhys. Lett. 23 (1993) 653.

[15] A. P. Gast, C. K. Hall, and W. B. Russel, J. Colloid Interface Sci. 96 (1983) 251.

[16] A. P. Gast, W. B. Russel and C. K. Hall, J. Colloid Interface Sci. 109 (1986) 161. 
[17] H. N. W. Lekkerkerker, W. C-K. Poon, P. N. Pusey, A. Stroobants and P. B. Warren, Europhys. Lett. 20 (1992) 559.

[18] D. Frenkel, R. J. Vos, C. de Kruif and A. Vrij, J. Chem. Phys. 84 (1986) 4625.

[19] M. P. Allen and D. J. Tildesley, Computer Simulation of Liquids (Oxford University Press, Oxford, 1987).

[20] E. J. Meijer and D. Frenkel, to be published. 